\title{
Tumour Angiogenesis and p53 Protein Expression in Mammary Phyllodes Tumors
}

Gary M.K . Tse, F.R.C.P.C., Philip C.W . Lui, M.B.,B.S., Richard A. Scolyer, F.R.C.P.A., Thomas C. Putti, Dip. Am. Bd., Fred Y.L . Kung, M.B.,Ch.B., Bonita K.B . Law, F.R.C.S., Tai-Shing Lau, Ph.D., C. Soon Lee, F.R.C.P.A.

Departments of Anatomical and Cellular Pathology (GMKT, FYLK) and Surgery (BKBL), Prince of Wales Hospital, Hong Kong; Department of Statistics, Chinese University of Hong Kong (T-SL), Hong Kong; Department of Pathology, United Christian Hospital (PCWL), Hong Kong; Department of Pathology, University of Sydney, and Department of Anatomical Pathology, Royal Prince Alfred Hospital, Sydney, Australia (RAS, CSL); and Department of Pathology, National University Hospital, Singapore (TCP)

We examined 186 phyllodes tumors (106 benign, 51 borderline, 29 malignant) for angiogenesis by assessing stromal microvessel density by the hot spot method and assessing p53 protein expression; we correlated these factors with stromal cellularity, margin status, nuclear pleomorphism, mitosis, and stromal overgrowth. Increased degree of malignancy in phyllodes tumors is associated with increased patient age and tumor size. Microvessel density and p53 protein expression also showed a similar increase with malignancy. Using a logistic regression model, microvessel density was shown to be useful in predicting malignancy in phyllodes tumors, independent of key criteria of stromal overgrowth, nuclear pleomorphism, and mitosis. Microvessel density showed correlation with stromal cellularity and margin status, suggesting an interrelationship between these parameters. P53 protein expression showed a positive correlation with microvessel density, suggesting possible overlap in the underlying mechanism of these two factors in the pathogenesis of phyllodes tumors. The numbers of recurrences and metastases are small in our series, and no significant difference was demonstrated in microvessel density and p53 protein expression compared with the primary. We conclude that microvessel density and p53 are useful as independent criteria in evaluating malignancy in phyllodes tumors.

Copyright (C) 2003 by The United States and Canadian Academy of Pathology, Inc.

VOL. 16, NO. 10, P. 1007, 2003 Printed in the U.S.A.

Date of acceptance: June 20, 2003.

Address reprint requests to: Gary M.K. Tse, Department of Anatomical and Cellular Pathology, Prince of Wales Hospital, Ngan Shing Street, Shatin, New Territory, Hong Kong, China; fax: 852-2637-4858; e-mail: garytse@cuhk.edu.hk.

DOI: $10.1097 / 01 . M P .0000089907 .67419 .42$
KEY WORDS: Angiogenesis, Microvessel density, Phyllodes tumor, p53.

Mod Pathol 2003;16(10):1007-1013

Mammary phyllodes tumor is an uncommon stromal epithelial neoplasm with a reported incidence of 0.3 to $0.5 \%$ of all breast lesions (1-5). It usually occurs in middle-aged women, but rarely it can occur in a younger age group and in men. Based on a combination of histologic criteria, including stromal cellularity, nuclear pleomorphism, mitotic rate, stromal overgrowth, and the margin status (whether infiltrative or rounded), phyllodes tumors can be divided into benign, borderline, and malignant groups $(1,6)$. Although all groups of phyllodes tumors show a propensity to recur locally, the borderline and malignant groups may also metastasize to other visceral organs.

Tumor angiogenesis as assessed by microvessel density has been under intensive investigation for its purported role as a prognostic factor in breast carcinoma. Many studies have demonstrated that tumor angiogenesis is related to various markers of tumor aggressiveness including grade, vascular invasion, lymph node metastases, and survival (7-13). Some studies have demonstrated the predictive utility of angiogenesis on outcome in node-negative breast carcinoma $(14,15)$ and in various stages of ductal carcinoma in situ (16). Other studies failed to confirm the relationship between tumor angiogenesis and other parameters of aggressiveness in breast carcinoma, or the outcome in ductal carcinoma $(11,17-19)$ or infiltrating lobular carcinoma $(20,21)$.

Several studies have addressed the relationship between angiogenesis and other nonhistologic prognostic markers, including p53 (22-25), proliferative indices such as Ki67 (23) and PCNA (26), apoptosis (26), cyclin D1 (24), CD44 (27), and laminin 
(27). Among these, p53 was the most widely studied, and most studies demonstrated an association between p53 and angiogenesis (22-24), although one study had conflicting results (25). The association of angiogenesis with other factors had been inconsistent.

The evaluation of angiogenesis in phyllodes tumors is limited to one previous study of 40 cases that showed increased microvessel density in borderline and malignant phyllodes tumors (28).

In this study, the authors investigated the angiogenesis of 186 phyllodes tumors collected from four institutions and correlated this with the histologic parameters of the phyllodes tumors, together with p53 protein expression.

\section{MATERIALS AND METHODS}

The histologic files of the four participating institutions were searched for instances of phyllodes tumors of the breast. The number of years searched ranged from 4 to 15 years. The paraffin blocks were retrieved, and $4-\mu \mathrm{m}$ slides were prepared routinely and stained with H\&E. All the slides were reviewed for the following histologic parameters: (1) stromal cellularity; (2) nuclear pleomorphism; (3) stromal overgrowth; (4) mitotic rate; and (5) margin of the tumor, whether infiltrative or rounded. Parameters 1 and 2 were graded as low/mild, moderate, or severe; stromal overgrowth was graded as mild, moderate (scanty epithelial elements within a lowpower field $(\times 40$, Nikon Labophot; field area, 1.9 $\mathrm{mm}^{2}$ ), or severe (absence of epithelial element within a low-power field), and the mitotic count was given as the number of mitotic figures per 10 high-power fields $(\times 400$, Nikon Labophot; field area, $0.19 \mathrm{~mm}^{2}$ ). A diagnosis of benign phyllodes tumor was made when there was low cellularity, no stromal overgrowth, mild pleomorphism, a rounded margin, and a mitotic count of two or less per 10 high-power fields. Malignant phyllodes tumor was diagnosed when the mitotic count was five or more per 10 high-power fields, together with stromal overgrowth and an infiltrative margin. Borderline phyllodes tumor was diagnosed when the histologic features were in between benignancy and malignancy.

For the microvessel density, one representative slide from each case was stained using an antibody to CD31 (1:50; DAKO, Glostrup, Denmark) with the avidin biotin method. The scoring of microvessel density was by the hot-spot method, with initial scanning of the slide at low power to identify the area with high vessel density; then 10 consecutive high-power fields $(\times 400$, Nikon, Labophot, field area $0.19 \mathrm{~mm}^{2}$ ) were examined, and any positively staining structures were counted, irrespective of whether a lumen was identified. Branching vessels were considered as one count. An average value was obtained as count per high-power field.

For p53 staining, the antibody used was monoclonal, reacting to both wild-type and mutant p53 (DO-7; Novocastra, United Kingdom) with microwave antigen retrieval. The staining of cells was assessed according to both the intensity and proportion of positive cells. The staining pattern was graded from 0 to 3 , with 0 being no staining; 1 , when $<33 \%$ of the stromal cell nuclei stained weakly; 2 , when $34-67 \%$ of cell nuclei stained with weak to moderate staining intensity; and 3 , when $>67 \%$ cells displayed moderate to strong nuclear staining.

For statistical analysis, PROC LOGISTIC in SAS was used to study the association between p53, microvessel density, and diagnosis with the presence of the five histologic factors and recurrence. Student's $t$ test and ANOVA was used to compare the tumor size, patient age, and diagnosis of phyllodes tumors. Statistical significance is established at $P<.05$.

\section{RESULTS}

\section{Clinicopathologic Findings}

A total of 186 cases of phyllodes tumors were retrieved. Among these, 106 were diagnosed as benign, 51 as borderline, and 29 as malignant. These phyllodes tumors were obtained from 180 patients, including 19 patients with one recurrence, 2 patients with two recurrences and 3 patients who had metastatic lesions ( 2 with distant organ metastases and 1 with intramammary seedings). This series of 186 phyllodes tumors included 14 first recurrences and 2 second recurrences.

All the patients were female, and their ages ranged from 14 to 77 years (mean, 42 y). One hundred ten patients were Chinese, 30 were Caucasian, 12 were Malaysian, 7 were Indian, 7 were Indochinese, and in 14 patients, the ethnicity was not known. Ninety-three phyllodes tumors were on the left, 90 were on the right, and in 3 , the side of lesions was not known. The sizes of the phyllodes tumors ranged from 0.8 to $22 \mathrm{~cm}$ (mean, $4.8 \mathrm{~cm}$ ).

For the 106 benign phyllodes tumors, patient age range was 17 to 62 years (mean, $40 \mathrm{y}$ ). The sizes of phyllodes tumors ranged from 0.8 to $22 \mathrm{~cm}$ (mean, $4 \mathrm{~cm}$ ). For the 51 borderline phyllodes tumors, patient age range was 20 to 77 years (mean, $45 \mathrm{y}$ ). The sizes of phyllodes tumors ranged from 1 to $20 \mathrm{~cm}$ (mean, $5.4 \mathrm{~cm}$ ). For the 29 malignant phyllodes tumors, patient age range was 35 to 76 years (mean, $47 \mathrm{y})$. The sizes of phyllodes tumors ranged from 1.5 to $22 \mathrm{~cm}$ (mean, $6.6 \mathrm{~cm}$ ). Between the benign and borderline groups, the differences in mean age and mean sizes of phyllodes tumors were significant $(P$ 
$<$.05), but the differences between the borderline and malignant groups were not. The results are summarized in Table 1.

\section{Microvessel Density}

For benign phyllodes tumors, the vessel count range was 2 to 25 (mean, 10); for borderline phyllodes tumors, the range was 5 to 23 (mean, 16); for malignant phyllodes tumors, the range was 14 to 37 (mean, 24; Figs. 1-2). The difference between the benign and borderline groups and between the borderline and malignant groups were statistically significant $(P<.0001)$.

Using a logistic regression model, microvessel density was useful in predicting the degree of malignancy even in the presence of histologic parameters of mitoses, nuclear pleomorphism, and stromal overgrowth. There was some overlap of information between the microvessel density and the other two parameters, stromal cellularity and margin status, in predicting the grade of phyllodes tumors so that these two parameters are not significant in the logistic regression model. The results are summarized in Table 1.

\section{Correlation between Microvessel Density and p53 Expression}

For the p53 score, the mean values were $0.69,0.9$, and 1.7, respectively for benign, borderline, and malignant phyllodes tumors (Fig 3). The results of p53 scores of some of the phyllodes tumors had been previously reported (29). The difference between the benign and borderline groups $(P<.05)$ and between the borderline and malignant groups $(P<.001)$ were also statistically significant.

When one considers the relationship between microvessel density and p53, the differences of the microvessel density values among the different p53 levels were significant using ANOVA. It was demonstrated that microvessel density and p53 show a positive correlation. Both microvessel density and p53 contribute in predicting diagnosis. There were no statistical differences of these parameters between the original and recurrent lesions. These results are summarized in Table 1.

\section{DISCUSSION}

This review of a large series of phyllodes tumors showed that benign, borderline, and malignant phyllodes tumors represent a histologic and demographic continuum as the patient age and tumor sizes gradually increase from benign to borderline to malignant. Furthermore, statistical significance can be established for differences in these parameters between the benign and borderline groups. However, for comparison between the borderline and malignant groups, statistical significance cannot be established, and the relatively small number of borderline and malignant phyllodes tumors probably accounts for this observation.

The evaluation of angiogenesis by microvessel density had been extensively studied in breast carcinoma. The methodologies used were varied, with the most commonly used methods being the hot spot method (30), as had been adopted in this study, the Chalkley method, or image analysis. Some investigators further suggested counting of blood vessels number per 1000 tumor cells (31). Several large-scaled studies had been carried out to compare the relative merits of these various methods, and both hot spot counting and the Chalkley method had been shown to correlate with other vascular parameters and prognostic estimates (32, 33). The advantages of using the hot spot method are its ease to perform, low cost, and relative speed of use. As a consequence, this method is still the most widely used. However, inherent in this simple but relatively effective method are several potential sources of error. As counting of microvessels is subjective, intra- and interobserver variations may be significant (33). Furthermore, it has been described that there existed marked heterogeneity of microvessel density in different parts of a carcinoma (34) as well as a relation between microvessel density and tumor volume (35). In phyllodes tumors, the most vascular areas (hot spots) were located around the epithelial component and tend to coincide with the high stromal cellularity and high mitotic activity areas. As the diagnosis of malignancy in phyllodes tumors is based on the highest cellularity and mitotic count, estimation of microvessel density may also be representative in relation to the entire lesion.

Table 1. Summary of the Number, Age, Size, Microvessel Density, and p53 Scores of the Three Categories of Phyllodes Tumors

\begin{tabular}{lcccc}
\hline & Number & $\begin{array}{c}\text { Age (years) } \\
\text { Mean (range) }\end{array}$ & $\begin{array}{c}\text { Tumor size (cm) } \\
\text { Mean (range) }\end{array}$ & $\begin{array}{c}\text { Microvessel Density } \\
\text { (vessels per high power fields): } \\
\text { mean (range) }\end{array}$ \\
\hline Benign & 106 & $40(17-62)$ & $4(0.8-22)$ & $10(2-25)$ \\
Borderline & 51 & $45(20-77)$ & $5.4(1-20)$ & $16(5-23)$ \\
Malignant & 29 & $47(35-76)$ & $6.6(1.5-22)$ & 0.69 \\
\hline
\end{tabular}



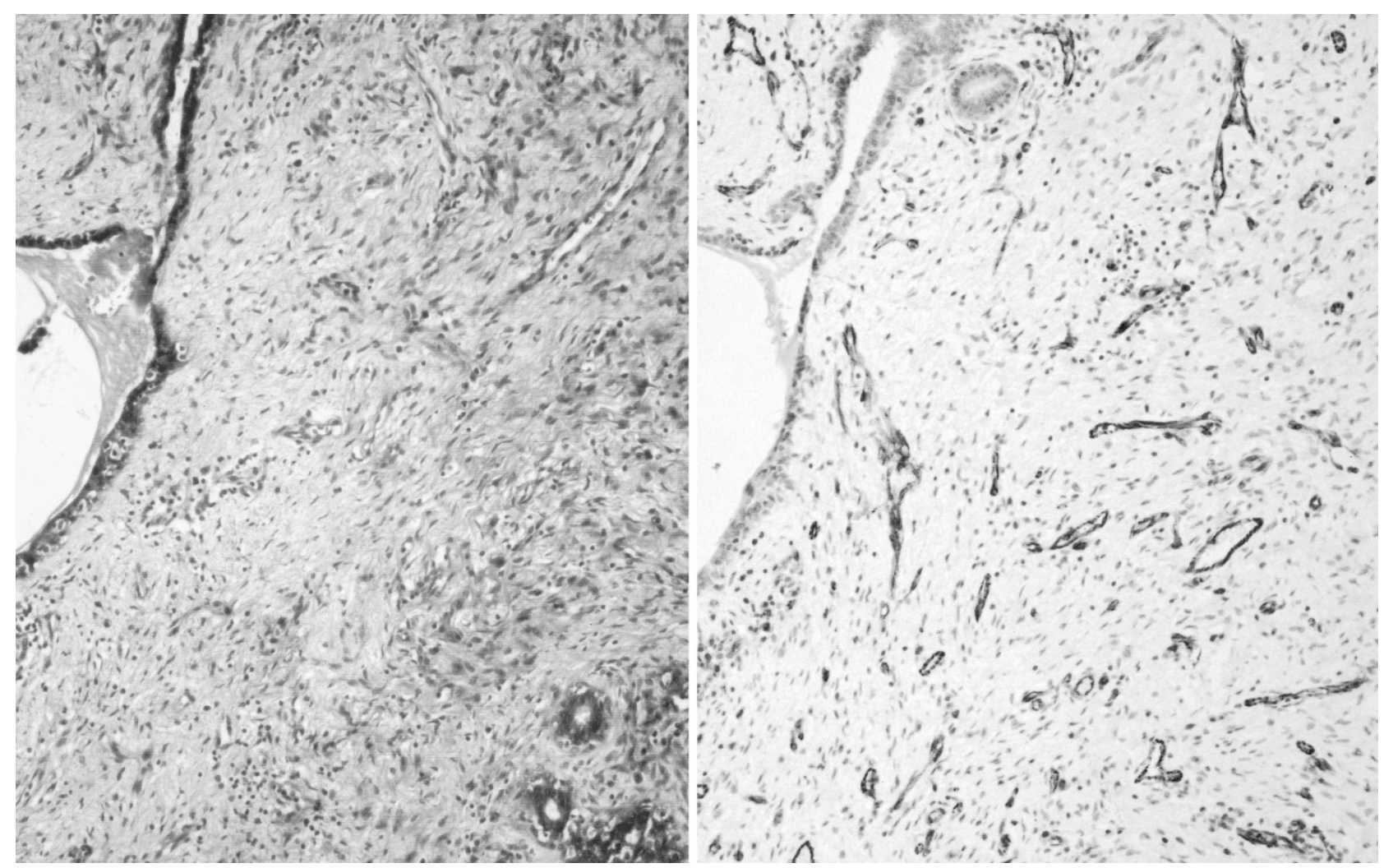

FIGURE 1. Photomicrograph showing a malignant phyllodes tumor (left panel, H\&E, $200 \times$ magnification) with high CD31 staining for microvessel density (right panel, $200 \times$ magnification).

The predictive role of microvessel density for the histologic parameters for malignancy and, hence, outcome appears to be variable and is affected by the type of the tumor. Although most studies were made on ductal carcinoma, two studies on infiltrating lobular carcinoma only showed higher microvessel density in infiltrating lobular carcinoma compared with infiltrating ductal carcinoma but failed to demonstrate any correlation of microvessel density with tumor histologic parameters (21) and overall survival (22).

Microvessel density appears to change during breast carcinogenesis. It has been reported in one study that microvessel density increased from ductal carcinoma in situ to early stromal invasion but decreased from early stromal invasive tumor to Tla and Tlb tumors, suggesting that increased vascularity may play a role in the early phases of transformation. In our series, the microvessel density showed a progressive rise from benign to borderline to malignant phyllodes tumors, suggesting that the role of microvessel density could be crucial in all stages of transformation of phyllodes tumors.

In our study, microvessel density was shown to be useful in predicting the degree of malignancy of phyllodes tumors, even in the presence of stromal cell mitoses, stromal nuclear pleomorphism, and stromal overgrowth, which are the key histologic criteria. This suggests that evaluation of stromal vascularity by assessing microvessel density may add information in the grading of phyllodes tumors. The correlation of microvessel density with stromal cellularity and to some extent margin status and the overlap of information by these parameters, as shown in the regression model, would indicate a strong interrelationship between stromal cellularity and microvessel density. This corresponds to the frequently made observation that cellular stromal areas are more vascular. Whether the increased vascularity is secondary to the stromal cellularity or vice versa remains to be elucidated, and this may involve locally released growth factors.

This observation is similar to that reported in breast carcinoma, especially for node-negative, early stage disease. Many studies have shown microvessel density to correlate with size, grade, vascular invasion, and early relapse, and microvessel density was inversely related to overall and relapsefree survival $(11,14,15,17,18)$. Other authors found microvessel density to be a powerful prognostic indicator in patient outcome independent of tumor grade, necrosis, mitotic index, and necrosis $(12,13)$. In metastatic ductal carcinoma, a few studies found microvessel density to correlate with the metastatic potential (36) and number of metastatic lymph nodes in high-grade carcinoma (31). Evaluation of the metastases showed that they had similar microvessel density compared with the primary tumor 


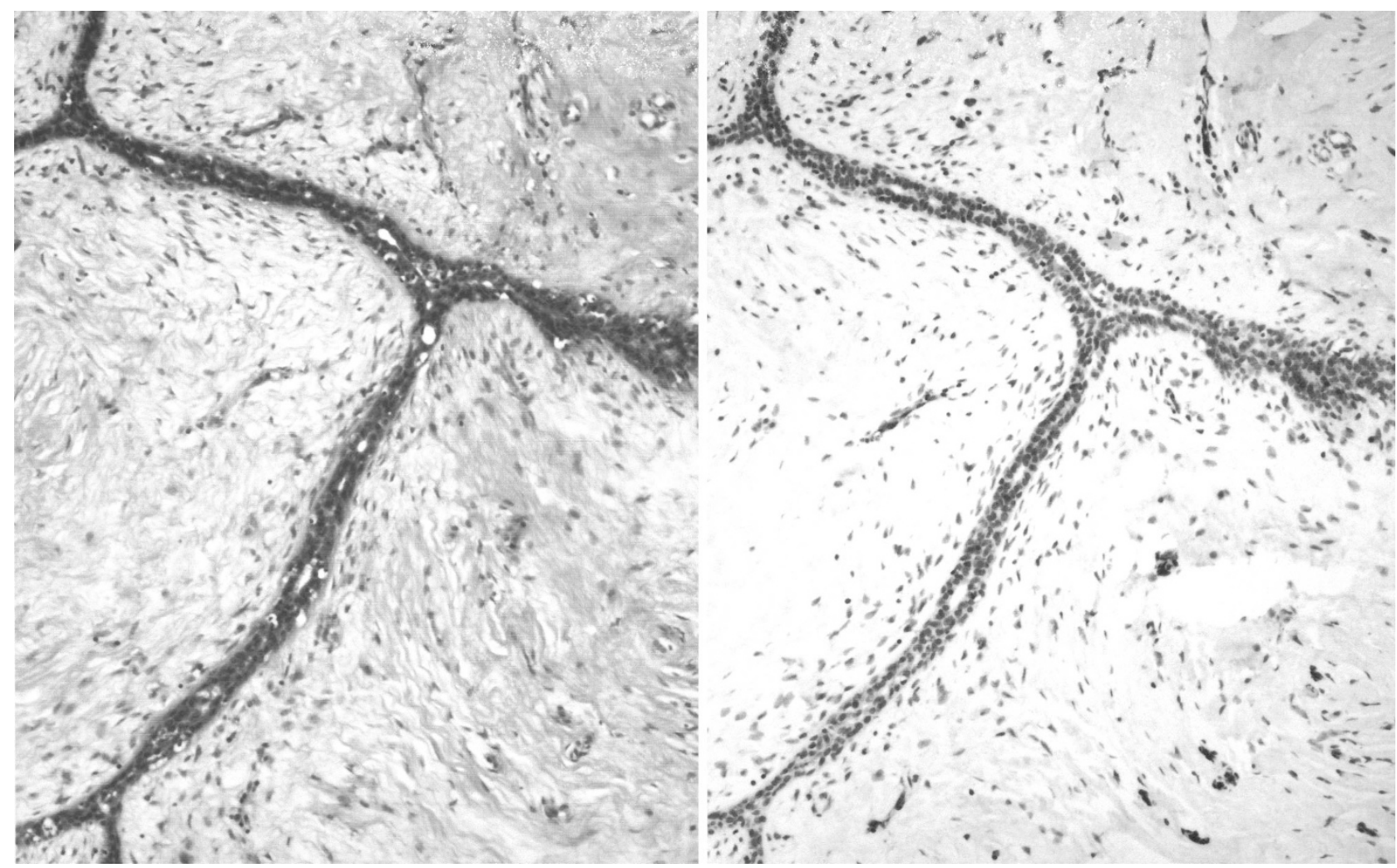

FIGURE 2. Photomicrograph showing a benign phyllodes tumor (left panel, H\&E, $200 \times$ magnification) with low CD31 staining for microvessel density (right panel, $200 \times$ magnification).
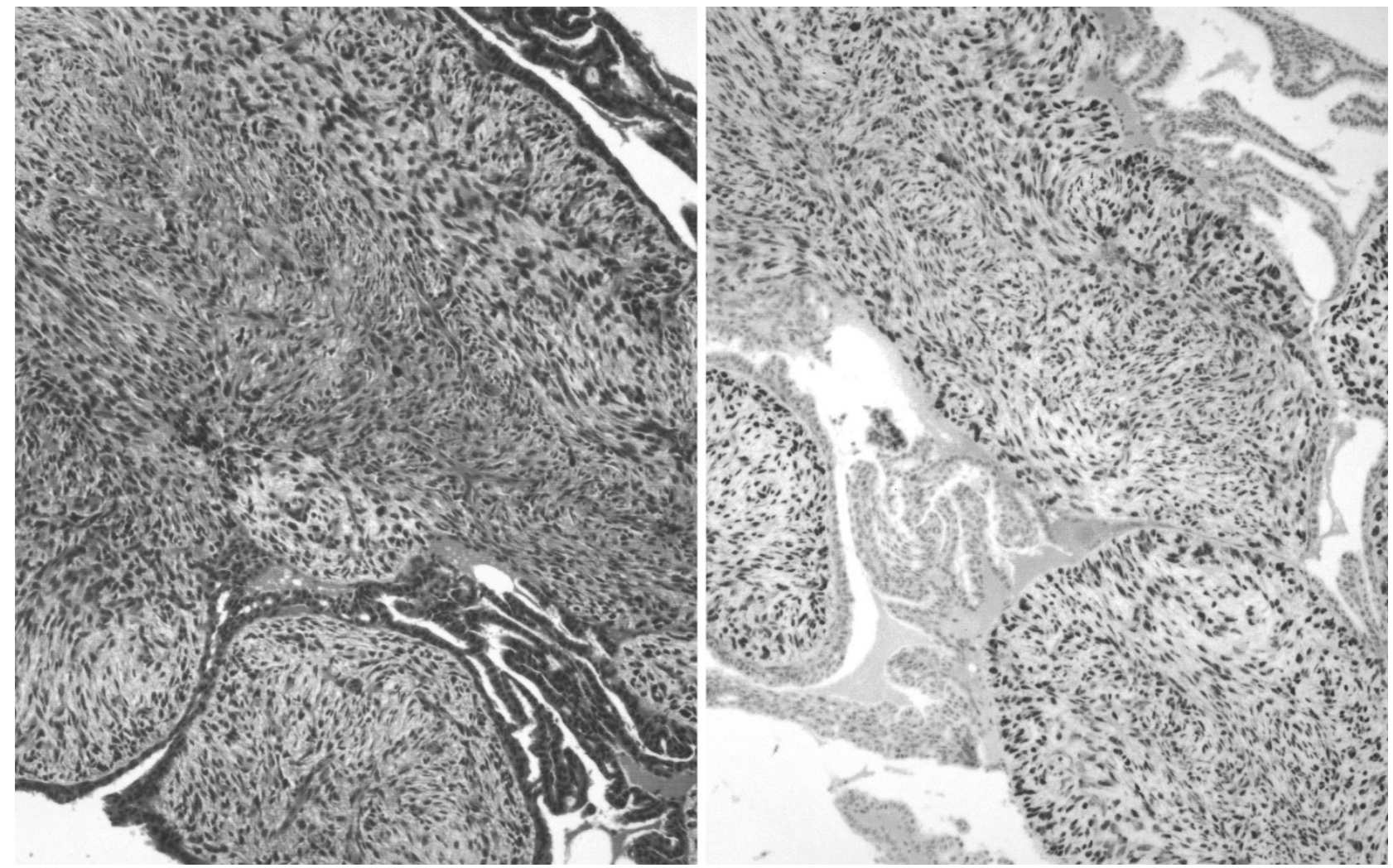

FIGURE 3. Photomicrograph showing a malignant phyllodes tumor (left panel, H\&E, 200× magnification) with strong positive p53 staining (right panel, $200 \times$ magnification). 
(37), and the level of vascularity in the metastases rather than that of the primary tumor was associated with decreased overall and relapse-free survival (38). The role of microvessel density in predicting visceral organ metastases was not clear, and in one study, no relationship was found (39).

In our series, the microvessel density did not show any demonstrable difference with the nonrecurrent phyllodes tumors within the same diagnostic category. Any predictive role of microvessel density in metastatic phyllodes tumors is even more problematic to assess as the number of cases with metastases was exceedingly small. To complicate the matter further, most metastases from phyllodes tumors are visceral and not to lymph nodes.

The relationship and prognostic value in patient outcome between p53 and microvessel density had been addressed in several studies in various other tumors including gastric carcinoma (40), colonic carcinoma and liver metastases (41), and ovarian cancers (42), in which a correlation of microvessel density and p53 had been demonstrated. In invasive and in situ ductal carcinoma, it was found that there was an association between microvessel density and p53 expression (22-24). Another study showed that both microvessel density and p53 were independent predictors of survival (25) in breast cancer. In this study, we found p53 to be positively correlated with microvessel density, and both were useful in predicting the degree of malignancy.

In conclusion, in this study, microvessel density as measured by the hot spot method showed significant increase with increasing degree of malignancy in phyllodes tumors. The highly significant difference in microvessel density between all three diagnostic groups of phyllodes tumors may indicate an important role of angiogenesis at both early and late stages of malignant progression. Furthermore, angiogenesis, like mitoses, stromal cell nuclear pleomorphism, and stromal overgrowth, is related to the grade of phyllodes tumors, suggesting that moderately or highly elevated microvessel density may be another useful parameter in assessing borderline and frank malignancy in phyllodes tumors. The higher the microvessel density and the expression of p53, the higher the degree of malignancy for the phyllodes tumor. The correlation of microvessel density and p53 suggests that they may be involved in the same mechanism in the pathogenesis of phyllodes tumors.

\section{REFERENCES}

1. Azzopardi JG. Sarcoma of the breast. In: Bennington J, editor. Problems in breast pathology. Vol II. Major problems in pathology. Philadelphia: Saunders; 1979. p. 355-9.

2. Palmer ML, De Risi DC, Pelikan A, Patel J, Nemoto T, Rosner $\mathrm{D}$, et al. Treatment options and recurrence potential for cystosarcoma phyllodes. Surg Gynecol Obstet 1990;170:193-6.

3. Kario K, Meada S, Mizuno Y, Makino Y, Tankawa H, Kitazawa S. Phyllodes tumor of the breast: a clinicopathologic study of 34 cases. J Surg Oncol 1990;45:46-51.

4. Rowell MD, Perry RR, Hsiu JG, Barranco SC. Phyllodes tumors. Am J Surg 1993;165:376-9.

5. Cole-Beuglet C, Soriano R, Kurtz AB, Meyer JE, Kopans DB, Goldberg BB. Ultrasound, X-ray mammography and histopathology of cystosarcoma phyllodes. Radiology 1983;146: 481-6.

6. Rosen PP. Breast pathology. 2nd ed. Philadelphia: Lippincott-Raven; 2000. p. 176-97.

7. Weider NW, Semple JP, Welch WR, Folkman J. Tumor angiogenesis and metastasis-correlation in invasive breast carcinoma. N Engl J Med 1991;324:1-8.

8. Horak E, Leek R, Klenk N, LeJeune S, Smith K, Stuart N, et al. Angiogenesis, assessed by platelet/endothelial cell adhesion molecule antibodies, as indicator of node metastasis and survival in breast cancer. Lancet 1992;340:1120-4.

9. Weidner N, Folkman J, Pozza F, Bevilacqua P, Allred EN, Moore DH, et al. Tumor angiogenesis: a new, significant, and independent prognostic indicator in early stage breast carcinoma. J Natl Cancer Inst 1992;84:1875-87.

10. Bosari S, Lee AKC, DeLellis RA, Wiley BD, Heatley GJ, Silverman ML. Microvessel quantitation and prognosis in invasive breast carcinoma. Hum Pathol 1992;23:755-61.

11. Vincent-Salomon A, Carton M, Zafrani B, Freneaux P, Nicolas A, Massemin B, et al. Long term outcome of small size invasive breast carcinomas independent from angiogenesis in a series of 685 cases. Cancer 2001;92:249-56.

12. Kato T, Kameoka S, Kimura T, Soga N, Abe Y, Nishikawa T, et al. Angiogenesis as a predictor of long-term survival for 377 Japanese patients with breast cancer. Breast Cancer Res Treat 2001;70:65-74.

13. de Jong JS, van Diest PJ, Baak JP. Hot spot microvessel density and the mitotic activity index are strong additional prognostic indicators in invasive breast cancer. Histopathology 2000;36:306-12.

14. Heimann R, Ferguson D, Powers C, Recant WM, Weichselbaum RR, Hellman S. Angiogenesis as a predictor of longterm survival for patients with node-negative breast cancer. J Natl Cancer Inst 1996;88:1764-9.

15. Karaiossifidi H, Kouri E, Arvaniti H, Sfikas S, Vasilaros S. Tumor angiogenesis in node-negative breast cancer: relationship with relapse free survival. Anticancer Res 1996;16: 4001-2.

16. Santinelli A, Baccarini M, Colanzi P, Fabris G. Microvessel quantitation in intraductal and early invasive breast carcinomas. Anal Quant Cytol Histol 2000;22:277-84.

17. Medri L, Nanni O, Volpi A, Scarpi E, Dubini A, Riccobon A, et al. Tumor microvessel density and prognosis in nodenegative breast cancer. Int J Cancer 2000;89:74-80.

18. Axelsson K, Ljung BM, Moore DH 2nd, Thor AD, Chew KL, Edgerton SM, et al. Tumor angiogenesis as a prognostic assay for invasive ductal breast carcinoma. J Natl Cancer Inst 1995;87:997-1008.

19. Van Hoef ME, Knox WF, Dhesi SS, Howell A, Schor AM. Assessment of tumour vascularity as a prognostic factor in lymph node negative invasive breast cancer. Eur J Cancer 1993;29A:1141-5.

20. Morphopoulos G, Pearson M, Ryder WD, Howell A, Harris M. Tumour angiogenesis as a prognostic marker in infiltrating lobular carcinoma of the breast. J Pathol 1996;180:44-9.

21. Nakopoulou L, Lekkas N, Lazaris AC, Athanassiadou P, Giannopoulou I, Mavrommatis J, et al. An immunohistochemical analysis of angiogenesis in invasive breast cancer with correlations to clinicopathologic predictors. Anticancer Res 1999;19:4547-53. 
22. Gonzalez-Palacios F, Sancho M, Martinez JC, Bellas C. Microvessel density, p53 overexpression, and apoptosis in invasive breast carcinoma. Mol Pathol 1997;50:304-9.

23. Zolota V, Gerokosta A, Melachrinou M, Kominea A, Aletra C, Scopa CD. Microvessel density, proliferating activity, p53 and bcl-2 expression in in situ ductal carcinoma of the breast. Anticancer Res 1999;19:3269-74.

24. Heffelfinger SC, Yassin R, Miller MA, Lower EE. Cyclin D1, retinoblastoma, p53, and Her2/neu protein expression in preinvasive breast pathologies: correlation with vascularity. Pathobiology 2000;68:129-36.

25. Tas F, Yavuz E, Aydiner A, Saip P, Disci R, Iplikci A, et al. Angiogenesis and p53 protein expression in breast cancer: prognostic roles and interrelationships. Am J Clin Oncol 2000;23:546-53.

26. Lee JS, Kim HS, Jung JJ, Kim YB, Park CS, Lee MC. Correlation between angiogenesis, apoptosis and cell proliferation in invasive ductal carcinoma of the breast and their relation to tumor behavior. Anal Quant Cytol Histol 2001;23:161-8.

27. Ozer E, Canda T, Kurtodlu B. The role of angiogenesis, laminin and CD44 expression in metastatic behavior of earlystage low-grade invasive breast carcinomas. Cancer Lett 1997;121:119-23.

28. Tse GM, Ma TK, Chan KF, Law BK, Chen MH, Li KH, et al. Increased microvessel density in malignant and borderline mammary phyllodes tumours. Histopathology 2001;38:56770 .

29. Tse GM, Putti T, Kung FY, Scolyer RA, Law BK, Lau TS, et al. Increased p53 protein expression in malignant mammary phyllodes tumors. Mod Pathol 2002;15:734-40.

30. Weidner N, Folkman J, Pozza F, Bevilacqua P, Allred EN, Moore DH, et al. Tumor angiogenesis: a new significant and independent prognostic indicator in early-stage breast carcinoma. J Natl Cancer Inst 1992;84:1875-87.

31. Laforga JB, Aranda FI. Angiogenic index: a new method for assessing microvascularity in breast carcinoma with possible prognostic implications. Breast J 2000;6:103-7.

32. Fox SB, Leek RD, Weekes MP, Whitehouse RM, Gatter KC, Harris AL. Quantitation and prognostic value of breast can- cer angiogenesis: comparison of microvessel density, Chalkley count, and computer image analysis. J Pathol 1995;177: 275-83.

33. Hansen S, Grabau DA, Rose C, Bak M, Sorensen FB. Angiogenesis in breast cancer: a comparative study of the observer variability of methods for determining microvessel density. Lab Invest 1998;78:1563-73.

34. Ahlgren J, Risberg B, Villman K, Bergh J. Angiogenesis in invasive breast carcinoma-a prospective study of tumour heterogeneity. Eur J Cancer 2002;38:64-9.

35. Wapnir IL, Barnard N, Wartenberg D, Greco RS. The inverse relationship between microvessel counts and tumor volume in breast cancer. Breast J 2001;7:184-8.

36. Leek RD. The prognostic role of angiogenesis in breast cancer. Anticancer Res 2001;21:4325-31.

37. Arapandoni-Dadioti P, Giatromanolaki A, Trihia H, Harris AL, Koukourakis MI. Angiogenesis in ductal breast carcinoma. Comparison of microvessel density between primary tumour and lymph node metastasis. Cancer Lett 1999;137: 145-50.

38. Guidi AJ, Berry DA, Broadwater G, Perloff M, Norton L, Barcos MP, et al. Association of angiogenesis in lymph node metastases with outcome of breast cancer. J Natl Cancer Inst 2000;92:486-92.

39. Paradiso A, Ranieri G, Silvestris N, Naccarato G, Bevilacqua G, Mangia A, et al. Failure of primary breast cancer neoangiogenesis to predict pattern of distant metastasis. Clin Exp Med 2001;1:127-32.

40. Huang SP, Wu MS, Shun CT, Wang HP, Lin JT. Tumor angiogenesis increases with nuclear p53 accumulation in gastric carcinoma. Hepatogastroenterology 2002;49:1453-6.

41. Kern A, Taubert H, Scheele J, Rudroff C, Mothes H, Kappler $\mathrm{M}$, et al. Association of p53 mutations, microvessel density and neoangiogenesis in pairs of colorectal cancers awnd corresponding liver metastases. Int J Oncol 2002;21:243-9.

42. Goodheart MJ, Vasef MA, Sood AK, Davis CS, Buller RE. Ovarian cancer p53 mutation is associated with tumor microvessel density. Gynecol Oncol 2002;86:85-90. 